\title{
The FGF system has a key role in regulating vascular integrity
}

\author{
Masahiro Murakami, ${ }^{1}$ Loc T. Nguyen, ${ }^{1}$ Zhen W. Zhang, ${ }^{1,2}$ Karen L. Moodie, \\ Peter Carmeliet, ${ }^{3}$ Radu V. Stan, ${ }^{4}$ and Michael Simons ${ }^{1,5}$

\begin{abstract}
${ }^{1}$ Angiogenesis Research Center and Section of Cardiology, Department of Medicine, and 2Department of Radiology, Dartmouth Medical School, Lebanon, New Hampshire, USA. ${ }^{3}$ Katholieke Universiteit Leuven, Leuven, Belgium. ${ }^{4}$ Department of Pathology, Dartmouth Medical School, Lebanon, New Hampshire, USA.
\end{abstract} \\ 5Department of Pharmacology and Toxicology, Dartmouth Medical School, Lebanon, New Hampshire, USA
}

\begin{abstract}
The integrity of the endothelial monolayer is essential to blood vessel homeostasis and active regulation of endothelial permeability. The FGF system plays important roles in a wide variety of physiologic and pathologic conditions; however, its role in the adult vasculature has not been defined. To assess the role of the FGF system in the adult endothelial monolayer, we disrupted FGF signaling in bovine aortic endothelial cells and human saphenous vein endothelial cells in vitro and in adult mouse and rat endothelial cells in vivo using soluble FGF traps or a dominant inhibitor of all FGF receptors. The inhibition of FGF signaling using these approaches resulted in dissociation of the VE-cadherin/p120-catenin complex and disassembly of adherens and tight junctions, which progressed to loss of endothelial cells, severe impairment of the endothelial barrier function, and finally, disintegration of the vasculature. Thus, FGF signaling plays a key role in the maintenance of vascular integrity.
\end{abstract}

\section{Introduction}

Vascular homeostasis is a complex process that includes growth of new vessels and maintenance of the existing vasculature. While the process of new vessel growth is beginning to be understood, relatively little is known about maintenance of the existing vasculature. In particular, while suppression of VEGF signaling results in regression of the tumor vasculature and certain types of normal fenestrated vasculature such as tracheal cartilage capillary network, most arterial and venous vasculature types do not seem affected, suggesting that a different growth factor might be involved $(1,2)$. We hypothesized that the FGF family may be involved in this process given FGFs ability to inhibit apoptosis and induce formation of tightly sealed capillaries $(3,4)$.

The FGF family consists of 22 structurally related polypeptide growth factors (5). Most FGFs are broad-spectrum mitogens and stimulate various cellular functions including migration, proliferation, and differentiation. These activities are critical to a wide variety of physiological as well as pathological processes including angiogenesis (6), vasculogenesis (7), wound healing (8), tumorigenesis (9), and embryonic development (10).

The FGFs produce their effects in target cells by signaling through cell-surface tyrosine kinase receptors. These FGF receptors (FGFRs) comprise 4 receptor tyrosine kinases designated FGFR1, FGFR2, FGFR3, and FGFR4. All are transmembrane proteins containing 2 or 3 extracellular Ig-like domains, a transmembrane domain, and an intracellular tyrosine kinase domain (11). Alternative splicing of the carboxyterminal half of the Ig domain III produces 2 to 3 isoforms (IIIa through IIIc) in all FGFRs, with the exception of FGFR4. While IIIb and IIIc isoforms are type I

Nonstandard abbreviations used: BAEC, bovine aortic endothelial cell; ECIS, electric cell-substrate impedance sensing; FGFR, FGF receptor; HSVEC, human saphenous vein endothelial cell; PV, pervanadate; RT, room temperature; SEM, scanning electron microscopy; sFGFR, soluble FGFR-IgCFc; ZO-1, zonula occludens-1.

Conflict of interest: The authors have declared that no conflict of interest exists.

Citation for this article: J. Clin. Invest. 118:3355-3366 (2008). doi:10.1172/JCI35298. transmembrane receptor tyrosine kinases, the IIIa splice variant encodes a truncated protein that cannot independently transduce extracellular signals. This alternative splicing event is regulated in a tissue-specific manner and dramatically affects ligand-receptor-binding specificity $(12,13)$.

Study of the biological role of the FGF system in adult tissues has been complicated by the great redundancy among FGFs and by an indispensable role played by Fgfr 1 and Fgfr2 in normal development, since the disruption of either gene leads to early embryonic death due to abnormal somite formation in the case of Fgfr 1 and the failure of early postimplantation development in the case of Fgfr2 (11). Thus, little is known regarding the role FGFs play in the adult vasculature. Recent studies have suggested that FGFR2 is involved in regulation of endothelial migration (14) and that signaling through myocardial FGFR1 and -R2 is important for coronary arterial development (15).

To investigate the role played by FGFs in the postdevelopmental stage, we elected to employ a systemic expression of soluble FGFRIgGFc (sFGFRs) chimeras capable of binding either extensive FGFs (sFGFR1IIIc and sFGFR3IIIc) or a limited subset of FGF family members (sFGFR3IIIb). Alternatively, we used a dominant negative construct (FGFR1DN) capable of inhibiting signaling of all 4 FGFRs (16). We then examined the effect of FGF signal silencing on the vasculature and on the endothelium per se in vivo as well as in vitro. We found that suppression of FGF signaling led to the loss of endothelial cell-cell contact due to decoupling of p120-catenin from VE-cadherin and subsequent disruption of adherens and tight junctions in both arteries and veins. This in turn resulted in increased vascular permeability and loss of vessel integrity. Thus, FGFs play a major role in the maintenance of vascular integrity in the existing adult vasculature.

\section{Results}

Inbibition of FGF signaling with sFGFR traps. To suppress FGF signaling in adult mouse vasculature, we employed adenovirusmediated systemic expression of sFGFRs (17) similar to a pre- 
A

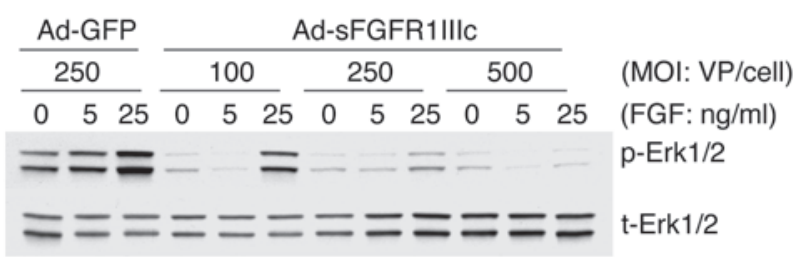

B

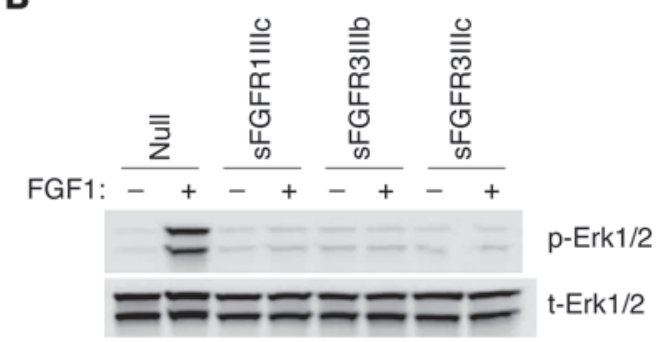

C

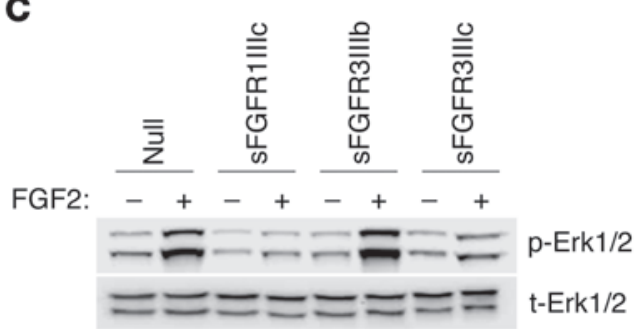

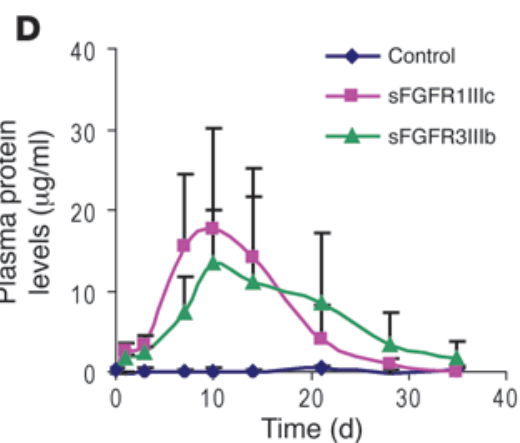
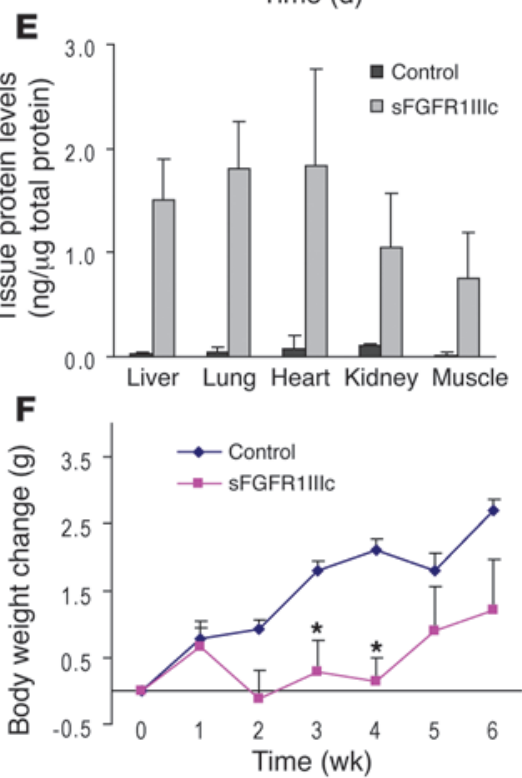

Figure 1

Validation of the sFGFR system. (A) sFGFR blocks FGF-induced Erk1/2 phosphorylation in vitro in a dose-dependent manner. Ad-GFP and Ad-sFGFR1Illc were transduced in BAECs and stimulated with indicated concentration of FGF1 for 10 minutes. Total cell lysates were subjected to Western blotting. p-Erk1/2, phospho-Erk1/2; t-Erk1/2, total Erk1/2; VP, viral particles. (B) sFGFRs effectively inhibit FGF1-induced Erk1/2 activation. Ad-Null, Ad-sFGFR1IIIc, Ad-sFGFR3IIIb, and Ad-sFGFR3IIlc were transduced in BAECs and stimulated with FGF1 for 5 minutes. (C) sFGFR3Illb does not inhibit FGF2-induced Erk1/2 activation. Ad-Null and Ad-sFGFRs were transduced in BAECs and stimulated with FGF2 for 5 minutes. (D) Plasma expression levels of sFGFR in mice. Ad-Null (control) or Ad-sFGFR1Illc $\left(5 \times 10^{10}\right.$ viral particles) was injected into C57BL/6 mice, and blood samples were taken at indicated time points. SFGFR levels were measured using an ELISA system detecting human IgG-Fc. Data are shown as mean $\pm \mathrm{SD}, n=4$ in each group. (E) Tissue distribution of sFGFR. After 10 days of Ad-Null (control) or Ad-sFGFR1Illc injection $\left(5 \times 10^{10}\right.$ viral particles), tissue samples were collected and total protein was extracted. Thereafter, ELISA assays detecting human IgGFc were performed. Data are shown as mean \pm SD, 4 animals in each group. (F) Body weight change after injection of sFGFR. Ten-week-old C57BL/6 mice were injected with adenoviruses $\left(5 \times 10^{10}\right.$ viral particles), and body weight was measured weekly. Data are shown as mean $\pm \mathrm{SD}$, $n=4$ in each group. ${ }^{*} P<0.05$, Student's $t$ test.

viously utilized anti-VEGF strategy (18). Three FGFR splicing isoforms were chosen: FGFR1IIIc, FGFR3IIIb, and FGFR3IIIc. FGFR1IIIc binds FGF1, $-2,-4,-5,-6$, and -8 , while FGFR3IIIb binds predominantly FGF1 and with lower affinity FGF9 and -20 . FGFR3IIIc has broad binding specificity to FGF1, -2,- 4, -8, -9, -17 , and -20 (13). The ability of these FGF traps to neutralize FGF activation was tested in bovine aortic endothelial cells (BAECs) following transduction with different amounts of adenoviruses encoding sFGFRs. A dose-dependent suppression of FGF1-induced Erk1/2 phosphorylation was observed in BAECs transduced with Ad-sFGFR1IIIc (Figure 1A). Whereas all sFGFRs inhibited FGF1-induced Erk1/2 phosphorylation, sFGFR3IIIb, which does not bind FGF2, did not inhibit FGF2induced Erk1/2 activation (Figure 1, B and C).
To achieve systemic expression of these traps in mice, adenoviruses encoding sFGFR1IIIc and sFGFR3IIIb constructs were injected i.v. into C57BL/ 6 mice, and circulating levels were determined using ELISA. High plasma levels of both constructs were detected starting 3-4 days after injection and were maintained for approximately 3 weeks (Figure 1D). Once expressed, sFGF traps exhibited uniform distribution and were detected in similar amounts in various mouse tissues (Figure 1E). Mice exposed to sFGFR1IIIc-Fc traps failed to gain weight while the traps were expressed, resuming weight gain when the expression declined (Figure 1F). This is consistent with a known role of angiogenesis in adipose tissue formation and maintenance (19). To minimize the possibility that inflammation/ immune responses elicited by adenoviruses may confound the outcome, we used the Fc construct with a mutation that eliminates 
A
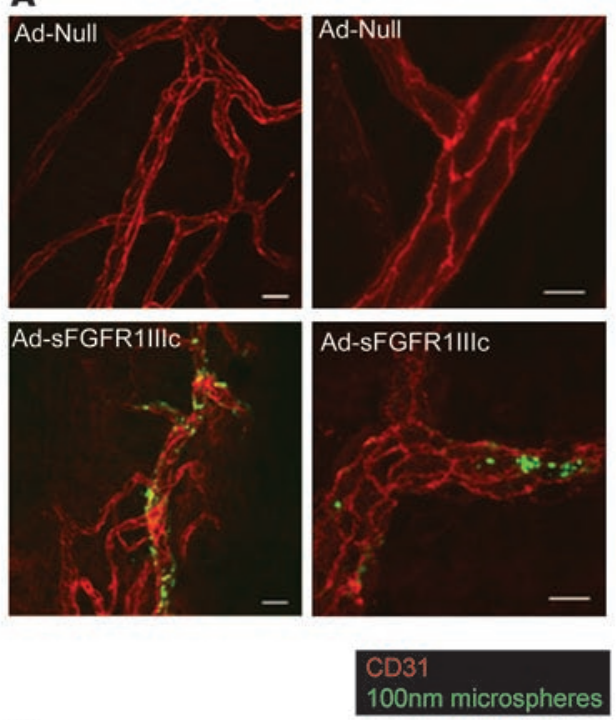

D
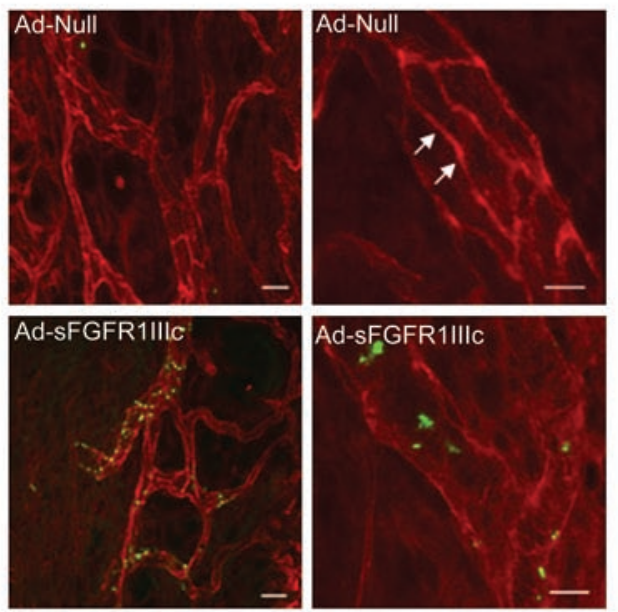

CD31

$100 \mathrm{~nm}$ microspheres
B

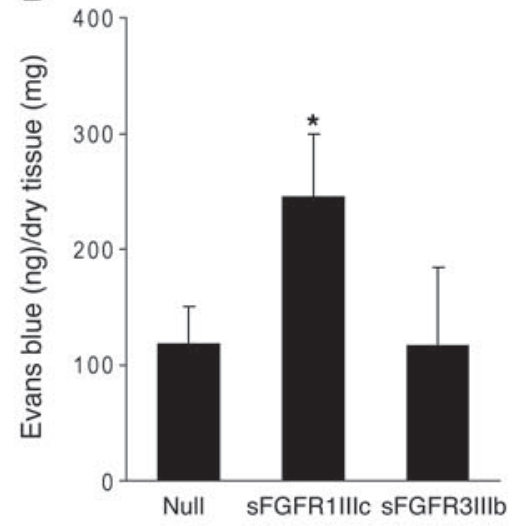

C

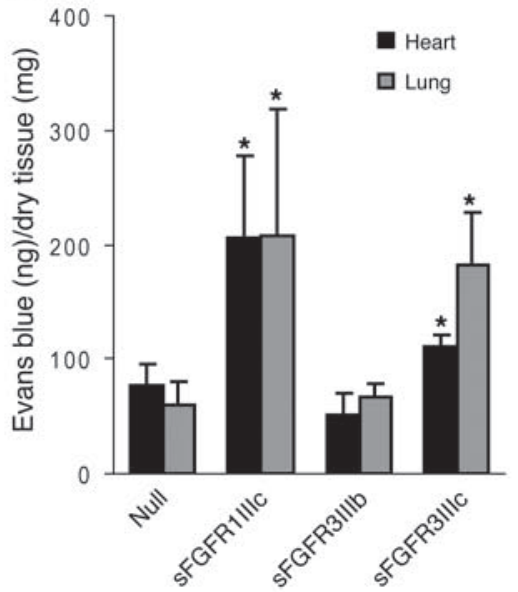

E
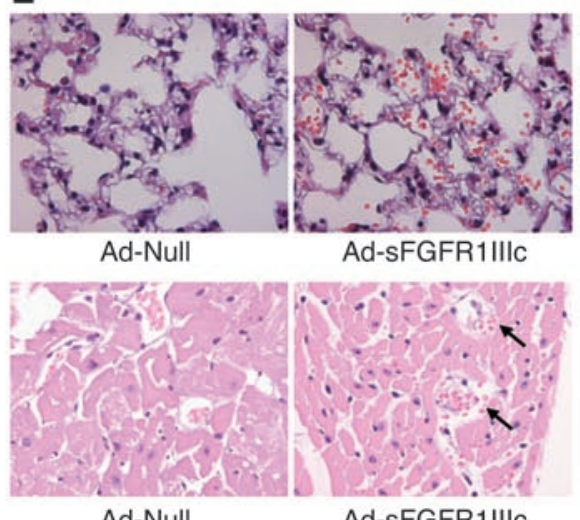

Ad-Null
Ad-sFGFR1IIIc

\section{Figure 2}

Impaired vascular integrity in mice lacking FGF signaling. (A) Increased vascular permeability and impaired endothelial morphology in sFGFR1IIIc mouse. Ten days after adenoviral injection in C57BL/6 mice, the trachea vasculature was stained for CD31 (red). Green represents microspheres in the extravascular space. Scale bars: $20 \mu \mathrm{m}$ (left panels); $10 \mu \mathrm{m}$ (right panels). (B) sFGFR1Illc increases vascular permeability in the muscle. Ad-Null (control) or Ad-sFGFR was injected in C57BL/6 mice, and 14 days later, Evans blue dye was injected i.v. The adductor group muscles were harvested for quantitation. Data are shown as mean $\pm \mathrm{SD}(n=4) .{ }^{*} P<0.05$, control vs. sFGFR1Illc by $t$ test. (C) Inhibition of FGF signaling in the heart and lung increases vascular permeability. Ad-Null (control), Ad-sFGFR was injected in C57BL/6 mice, and 10 days later, Evans blue dye levels were examined in the heart and lung. Data are shown as mean \pm SD for $n=7$, control; $n=8$, sFGFR1IIlc; $n=4$, sFGFR3IIlb; $n=4$, sFGFR3IIIc. ${ }^{*} P<0.05$, vs. control by $t$ test. (D) Increased vascular permeability in Ad-sFGFR1Illc-treated nude mouse. NU/NU nude mice (Charles River Laboratories) were subjected to trachea CD31. Junctional CD31 staining observed in control (arrows) is completely absent in the Ad-sFGFR1Illc-treated trachea vasculature. Scale bars: $20 \mu \mathrm{m}$ (left panels); $10 \mu \mathrm{m}$ (right panels). (E) Hemorrhage observed in Ad-sFGFR1Illctreated mouse lung and heart. Ten days after adenoviral injection in nude mice, lung and heart were harvested for H\&E staining. Upper panels show lung sections and lower panels show heart sections. Black arrows indicate myocardial hemorrhage. Original magnification, $\times 400$.

Fc receptor binding (20). Moreover, nude mice injected with either null or sFGFR1IIIc adenoviruses had no change in body weight (Ad-Null, control, $33.94 \pm 1.03$ g; sFGFR1IIIc, $32.88 \pm 0.48$ g at 8 weeks) despite high plasma levels of sFGFR1IIIc $(64.2 \pm 18.3 \mu \mathrm{g} / \mathrm{ml}$ at week 4) that persisted for at least 8 weeks $(25.8 \pm 5.9 \mu \mathrm{g} / \mathrm{ml}$ at week 8). This strongly argues that an inflammatory response, if any, had no effect on impact seen with the other sFGFR traps.
Disruption of endothelial FGF signaling affects vessel integrity and permeability. The tracheal vasculature is known to be disrupted by a systemic expression of circulating VEGF traps (1). Following systemic expression of sFGFR1IIIc, we observed a loss of vascular integrity in the tracheal circulation as demonstrated by extravasation of $100 \mathrm{~nm}$ i.v.-injected microsphere particles that was not present in mice exposed to the control adenovirus (Figure 2A). To further 
A
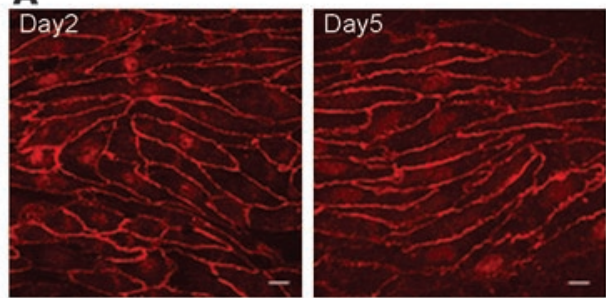

Ad-Null

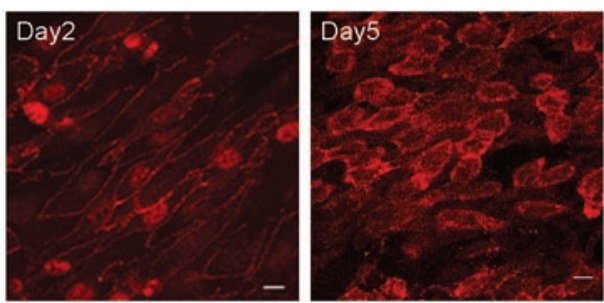

Ad-FGFR1DN
B
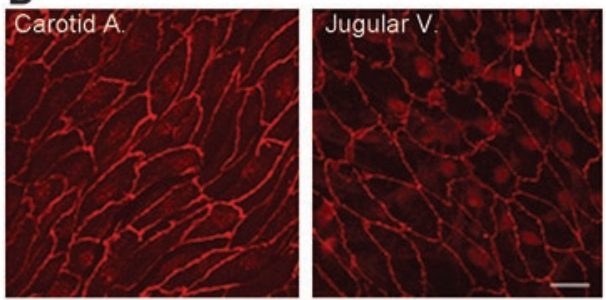

Ad-Null
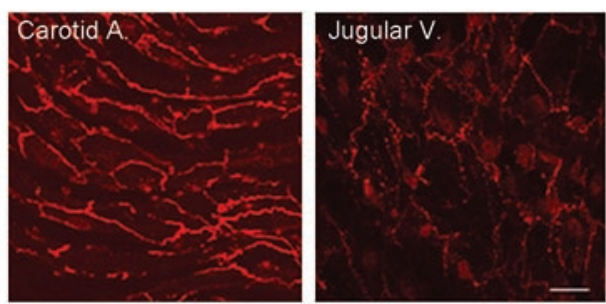

Ad-sFGFR1IIIc

\section{Figure 3}

In vivo effect of FGF inhibition in the endothelium. (A) Enface preparation of the rat femoral artery transduced with Ad-Null or Ad-FGFR1DN (109 PFUs). Segments of arteries were transduced with adenoviruses and stained for VE-cadherin (red) and maximum intensity projection of $1-\mu \mathrm{m}$ Z-Stack sections is shown. At day 5 , endothelial cells lost cell-cell contacts and gaps formed between cells. Scale bars: $10 \mu \mathrm{m}$. (B) Mouse carotid artery and jugular vein exposed to systemic Ad-Null or Ad-sFGFR1IIIc viruses. Segments of arteries were stained for VE-cadherin, and maximum intensity projection of $1-\mu \mathrm{m}$ Z-Stack sections is shown. Scale bars: $20 \mu \mathrm{m}$. characterize this loss of vascular integrity, we evaluated vascular permeability in normal C57BL/ 6 mice following systemic expression of either sFGFR1IIIc, sFGFR3IIIb, or a control construct. In agreement with the observed loss of tracheal vasculature integrity, there was a substantial increase in Evans blue dye retention in the skeletal muscle in mice exposed to sFGFR1IIIc but not sFGFR3IIIb virus and control mice (Figure $2 \mathrm{~B}$ ). The same Evans blue assay demonstrated a significant increase in permeability in both myocardial and pulmonary tissue in sFGFR1IIIc- and sFGFR3IIIcexposed but not control or sFGFR3IIIb mice (Figure 2C).

To assess the effect of prolonged suppression of FGF signaling, nude mice were injected with Ad-FGFR1IIIc-Fc. After 8 weeks of exposure of sFGFR1IIIc trap, the mortality rate was $15.3 \%$, unlike C57BL/6 mice, which showed very little mortality. Histological examination showed disrupted vascular integrity in the tracheal circulation (Figure 2D) and scattered areas of arterial thrombosis and hemorrhage in the lungs and heart (Figure 2E).

To obtain an insight into the cause of this loss of vascular integrity, arterial endothelium of a normal rat femoral artery was exposed in vivo to either the Ad-FGFR1DN or Ad-Null virus. FGFR1 dominant negative construct (FGFR1DN), a cytoplasmic truncated form of mouse FGFR1IIIc, is capable of suppressing signaling of all 4 FGFRs by heterodimerizing with other FGFR isoforms $(16,21)$.

Two days after viral transduction, enface VE-cadherin staining demonstrated normal endothelial morphology in the artery exposed to Ad-Null, while there was a loss of endothelial cell-cell contacts in the artery exposed to FGFR1DN (Figure 3A). Five days later, the majority of endothelial cells in the Ad-FGFR1DN-exposed artery appeared round and not in contact with each other, indicating a further loss of cell-cell contact (Figure 3A). Remarkably, endothelial cells were no longer aligned along the axis of the blood flow, suggesting the loss of either the ability to sense the shear stress or to respond to it. Similar findings, albeit somewhat less dramatic, were observed both in the arterial and venous systems of mice with systemic expression of the sFGFR1IIIc-Fc trap, suggesting that the FGF inhibition effect is not restricted to the arterial vasculature (Figure 3B).

Scanning electron microscopy (SEM) confirmed the loss of cellcell contacts (Figure 4B). Furthermore, several areas of the arterial segment exposed to FGFR1DN demonstrated a complete loss of endothelial lining, leading to the exposure of the underlying basement membrane and platelet adhesion (Figure 4B). To further evaluate the appearance of endothelial junctions, we employed transmission electron microscopy. Whereas endothelial junctions exposed with control virus were tightly sealed (Figure 4C), FGFR1DN-transduced endothelium demonstrated open junctions and detachment of endothelial cells (Figure 4, D-F)

To further examine the effect of suppression of endothelial FGF signaling on permeability, we carried out in vitro studies using both FGFR1DN and sFGFRs. Ad-FGFR1DN transduction of BAEC or exposure of these cells to sFGFR1IIIc or sFGFR3IIIc trap resulted in a significant decrease in transendothelial impedance compared with control cells 48 hours later (Figure 5, A and B). Furthermore, the Ad-FGFR1DN-transduced monolayer was permeable to $70 \mathrm{kDa}$ and $2000 \mathrm{kDa}(2 \mathrm{MDa})$ dextran, suggesting the presence of large gaps in the monolayer (Figure 5C). Exposure of the mature BAEC monolayer to sFGFR1IIIc but not FGFR3IIIb traps also significantly increased permeability to $70 \mathrm{kDa}$ and 2 MDa dextran (Figure 5D).

FGF signaling is required for maintenance of adherens junctions. The 2 types of junctions found in the endothelium and contributing to structural stability are adherens and tight junctions (22). Many reports support the idea that tight junction organization is largely dependent on the formation of adherens junctions. In some cellular systems, blocking adherens junctions inhibits the correct organization of tight junctions (23). Adherens junctions play a critical role not only in regulation of vascular permeability but also in maintenance of vessel stability (24). Since VE-cadherin is the principal protein regulating formation of adherens junctions in endothelial cells, we evaluated its expression in Ad-FGFR1DN-transduced endothelial cells in vitro. BAEC monolayers were transduced when fully confluent at an MOI ensuring approximately $30 \%-50 \%$ transduction to avoid possible toxic adenoviral effects. In cells expressing the dominant negative construct, regular VE-cadherin staining at adherens junctions at the cell periphery showed interruptions that correlated with gaps in the monolayer by phase contrast or differential interference contrast. No such changes were observed in Ad-GFP-transduced cells (Figure 6A). Exposure to the Ad-FGFR1IIIc-Fc-encoded trap led to a similar loss of junctional VE-cadherin expression (Figure 6B). 
A
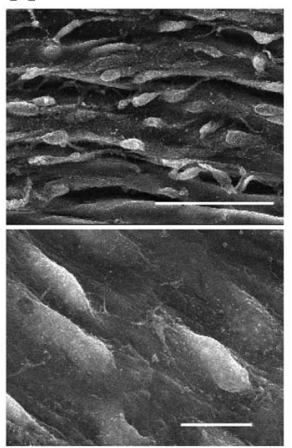

D
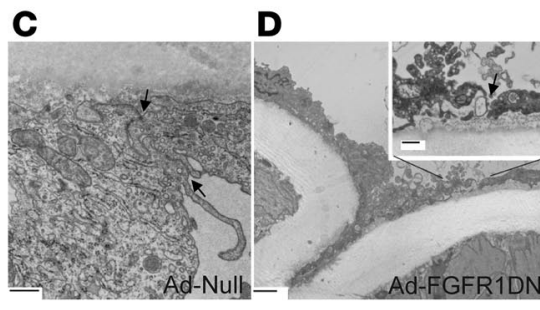

E
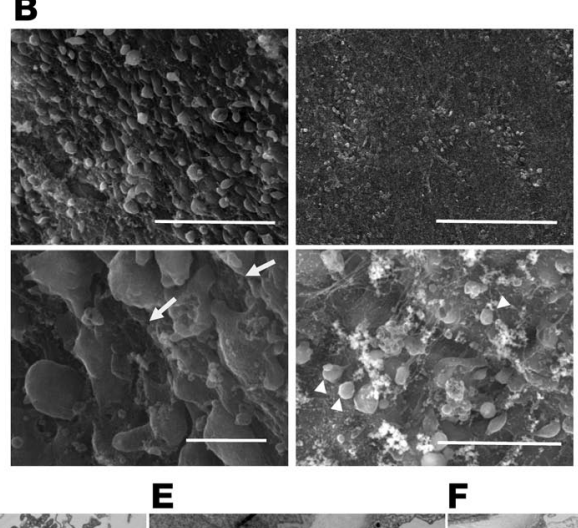

$\mathbf{F}$

\section{Figure 4}

Effect of FGF inhibition in the endothelium: electron microscopy analyses. (A) SEM analysis of rat femoral arteries transduced with Ad-Null. The arterial wall shows ridges formed due to contractions in the underlying smooth muscle cells (top panel, scale bar: $50 \mu \mathrm{m}$ ). Higher magnification detail of the Ad-Null-infected artery demonstrates the continuous endothelial monolayer (bottom panel, scale bar: $10 \mu \mathrm{m}$ ). (B) SEM analysis of Ad-FGFR1DN-transduced rat femoral artery. The area with remaining endothelial cells is shown (left panels, scale bars: $50 \mu \mathrm{m}$ [top]; $10 \mu \mathrm{m}$ [bottom]). Endothelial cells are swollen and have lost contacts with neighboring cells. Underlying matrix layer is partially exposed (white arrows). Area demonstrating severe loss of the endothelium with exposure of the subjacent basement membrane is also shown (right panels, scale bar: $50 \mu \mathrm{m}$ [top]). Higher magnification shows a few cells, presumably platelets by size, adhering to the vessel wall (white arrowheads) (right panels, scale bar: $10 \mu \mathrm{m}$ [bottom]). (C) Transmission electron microscopy analysis of Ad-Null-transduced rat femoral artery (day 2). Adjacent endothelial cells form a tightly sealed junction indicated by black arrows. Scale bar: $500 \mathrm{~nm}$. (D) Ad-FGFR1DN-transduced artery (day 1). Scale bar: $2 \mu \mathrm{m}$. Inset: a gap is formed between endothelial cells. Arrow (inset) indicates open cell-cell junction. Scale bar: $500 \mathrm{~nm}$. (E) Ad-FGFR1DN-transduced artery (day 2) shows an open endothelial junction (black arrows). A platelet (PLT) adheres to the open space and extends a cellular process (white arrowhead). Scale bar: $500 \mathrm{~nm}$. (F) Ad-FGFR1DN-transduced artery showing a wide gap in the endothelium (black arrow) and an EC detaching from the basement membrane. Scale bar: $2 \mu \mathrm{m}$.

The same cells also demonstrated a loss of tight junctions as demonstrated by zonula occludens-1 (ZO-1) staining (Figure 7A). The effect of suppression of FGF signaling on cell membrane VE-cadherin expression was not limited to the arterial endothelium, as transduction of human saphenous vein endothelial cells (HSVECs) with Ad-FGFR1DN demonstrated a similar loss of VE-cadherin from cell-cell junctions (Figure 7B). Furthermore, quantitative analysis of FGFR1DN-transduced cells confirmed visual observations. The number of cells with interrupted junctional VE-cadherin staining increased as the viral dose of Ad-FGFR1DN but not Ad-GFP increased, while overall cell density remained unaltered, suggesting that the effect of FGF signaling disruption on VE-cadherin expression is not due to cell loss (Supplemental Figure 1, A and B; supplemental material available online with this article; doi:10.1172/JCI35298DS1). Moreover, Western blotting demonstrated no increase in caspase 3 cleavage in FGFR1DN-expressing endothelial cells compared with controls (Supplemental Figure 1D).
In order to examine these cells more closely, we employed SEM to examine Ad-GFP- and Ad-FGFR1DN-transduced endothelial monolayers in vitro. Ad-GFP-expressing monolayer appeared continuous with rare gaps that normally occur in endothelial cells in culture (Supplemental Figure 1C). Conversely, Ad-FGFR1DN-expressing monolayers demonstrated a sharp increase in the number and size of gaps within the monolayer (Supplemental Figure 1C) as soon as 24 hours after transduction that was then followed by a progressive disassembly of the monolayer. Since the data presented above suggested that FGFs serve to protect vascular integrity, we measured endothelial cell monolayer permeability in vitro after FGF and VEGF exposure. In agreement with previous results (25), exposure to VEGF-A markedly decreased monolayer impedance. In contrast, exposure to FGF1 slightly but significantly increased impedance (and thus reduced permeability) (control, $1652.68 \pm 58.3$ ohms; FGF1: $1776.08 \pm 48.62$ ohms at 8 hours, $P<0.05)$. Furthermore, pretreatment with low concentration of FGF prevented VEGF-induced increase of monolayer permeability (Figure 8A). Moreover, while under conditions in which a confluent endothelial monolayer was maintained without serum supplementation, VEGF-A rapidly disassembled adherens junctions as demonstrated by movement of VE-cadherin from cell-cell contacts into the cytosol (Figure $8 \mathrm{~B})$. However, such an effect was not observed with FGF1 treatment (Figure 8B). Thus, these experiments demonstrate that FGF signaling regulates endothelial permeability and vascular integrity in vitro and in vivo.

FGF inhibition disrupts VE-cadherin-based junctional complex. To further analyze VE-cadherin expression, the presence of plasma membrane and total cell VE-cadherin was assessed by Western blotting of Ad-FGFR1DN- and Ad-GFP-transduced BAECs following cell surface biotinylation. The Ad-FGFR1DN BAECs demonstrated significant loss of plasma membrane VE-cadherin (Figure 9A) even though total cell expression of VE-cadherin or other adherens junction proteins such as $\beta$-catenin and $\mathrm{p} 120$ catenin as well as tight junction protein $\mathrm{ZO}-1$ were unchanged (Figure 9B). There was increased cytoplasmic VE-cadherin staining in Ad-FGFR1DN cells, suggesting VE-cadherin redistribution to the cytosol (Supplemental Figure 1B).

The expression of $\mathrm{N}$-cadherin, which is known to control VE-cadherin levels in endothelial cells, was not changed either in the biotinylated (cell surface) or total protein fraction in FGFR1DN cells (Figure 9A) (26). Furthermore, endothelial cell adhesion to smooth muscle cells, thought in part to be mediated by N-cadherin, was not affected by FGF inhibition in vitro (Supplemental Figure 2A). Finally, subendothelial distribution of $\mathrm{N}$-cadherin was unchanged in the vasculature exposed to sFGFR1IIIc adenovirus (Supplemental Figure 2B). 


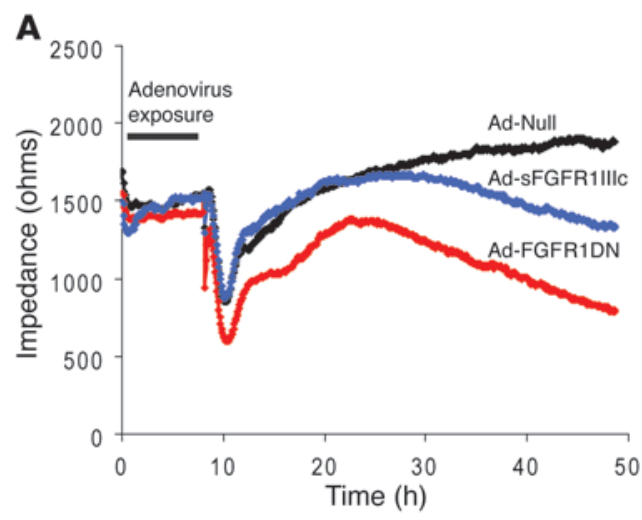

B

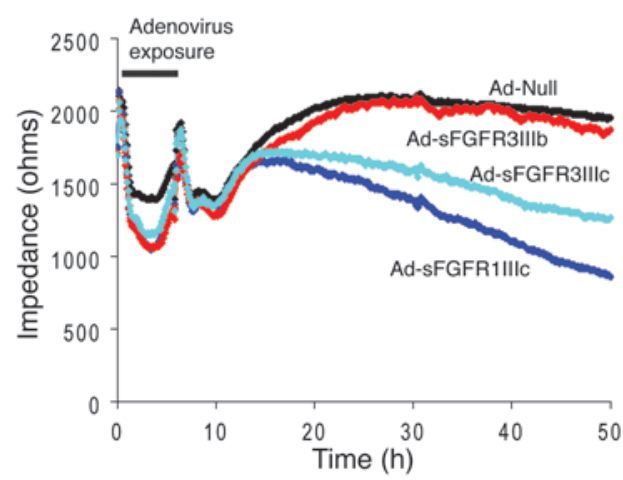

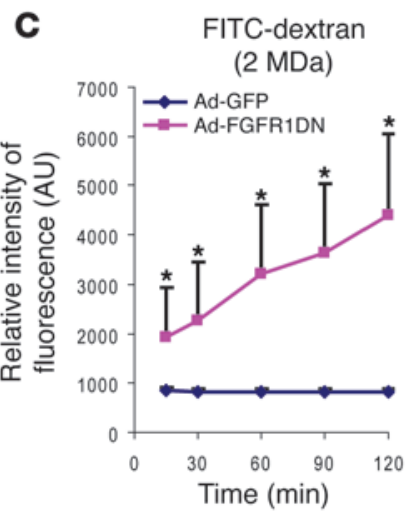

D

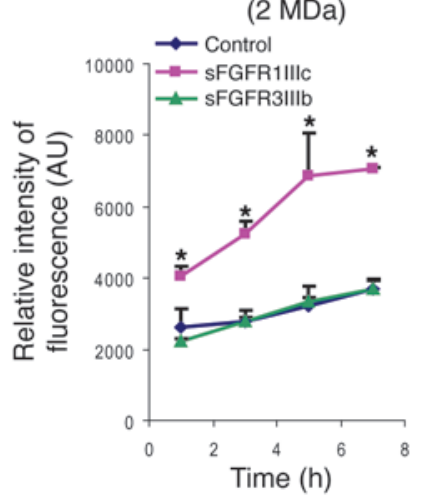

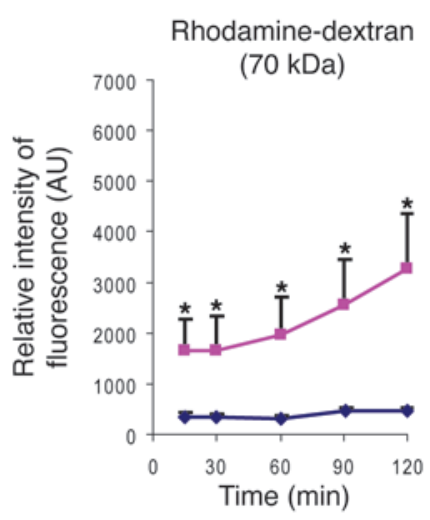

Rhodamine-dextran

$(70 \mathrm{kDa})$

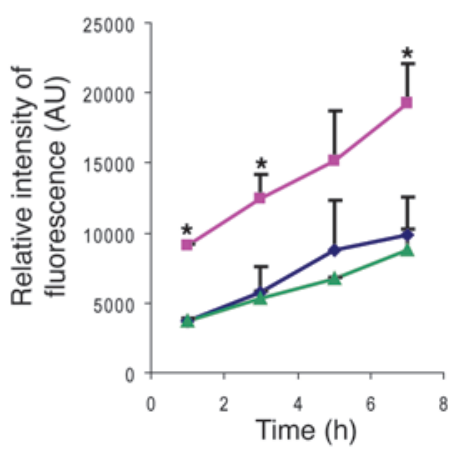

Figure 5

Increased endothelial permeability in cells lacking FGF signaling. (A) Endothelial monolayer permeability evaluated with ECIS system. Impedance was measured every 5 minutes for 48 hours after the onset of adenoviral transduction. After adenoviral exposure, cell culture medium was changed to normal growth medium. $n=3$ in each group, $P<0.05$, control versus FGFR1DN or sFGFR1Illc at 48 hours (B) ESIS analysis using various sFGFR adenoviruses. After adenoviral exposure, medium was replaced with normal growth medium. $n=3$ in each group, $P<0.05$, control versus sFGFR1IIlc or sFGFR3IIlc at 48 hours (C) Transwell tracer experiment using high (2 MDa) and low (70 kDa) molecular weight dextran. Full confluent BAECs on the Transwell chambers were transduced and dextrans were added in the upper chamber. Fluorescent values in the lower chamber were measured at indicated time points using a fluorescent microplate reader. Three independent experiments were performed. One representative experiment is shown as mean \pm SD for $n=3$ in each group; ${ }^{\star} P<0.05$ by Student's $t$ test, control vs. FGFR1DN. AU represents relative intensity of fluorescence. (D) Transwell tracer experiment using Ad-sFGFRs. Data are shown as mean \pm SD for $n=3$ in each group; ${ }^{*} P<0.05$ by Student's $t$ test, control vs. sFGFR1IIIc.

VE-cadherin/p120-catenin binding plays an important role in retention of VE-cadherin at cell-cell contacts and maintenance of vascular integrity (27). Treatment with FGF1 resulted in increased VE-cadherin/p120-catenin association as demonstrated by VE-cadherin immunoprecipitation followed by Western blotting with an anti-p120-catenin antibody (Figure 9C). In contrast, expression of the FGFR1DN construct led to a marked decrease in VE-cadherin/ p120-catenin association while VE-cadherin/ $\beta$-catenin association was not disrupted (Figure 9D). A similar result was observed following BAEC treatment with tyrosine phosphatases inhibitor pervanadate (PV) (Figure 9D). Thus, VE-cadherin stability, which is known to be mediated by VE-cadherin-p120-catenin complex formation, is affected by the shutdown of FGF signaling.

\section{Discussion}

By using complimentary approaches to shut down endothelial FGF signaling in various physiologic settings and different vascular beds, we observed dissolution of VE-cadherin/p120-catenin complex and loss of endothelial adherens and tight junctions in vitro and in blood vessels in vivo. This in turn led to increased permeability and tissue hemorrhage due to the loss of vascular integrity. Thus, continuous FGF signaling appears to play a critical role in the maintenance of vascular integrity and regulation of basal permeability. Furthermore, our study demonstrates that the maintenance of vascular integrity is a dynamic process, requiring active signaling events.

Although FGFRs are essential for normal development, the role played by the FGF/FGFR system in adult vasculature has not been studied due to early lethality of Fgfr1 and Fgfr2 knockout mice (11) and great redundancy of the FGF family (5). To overcome these obstacles, we employed 3 FGF traps that effectively inactivate either a wide (FGFR1IIIc-Fc and FGFR3IIIc) or a limited (FGFR3IIIb-Fc) range of FGF family members. In addition to providing an alternative to FGFR1DN-dependent inactivation of FGF signaling, traps allow differentiation of contribution from different FGFs to endothelial signaling.

Both FGFR1DN and sFGFR1IIIc induced similarly severe effects including loss of endothelial cell-cell contact and marked increase in permeability. The effects were considerably less pronounced with the FGFR3IIIb trap, implying that FGF1, -9 , and -20 by themselves are not critical to ongoing vascular integrity maintenance or that 
A

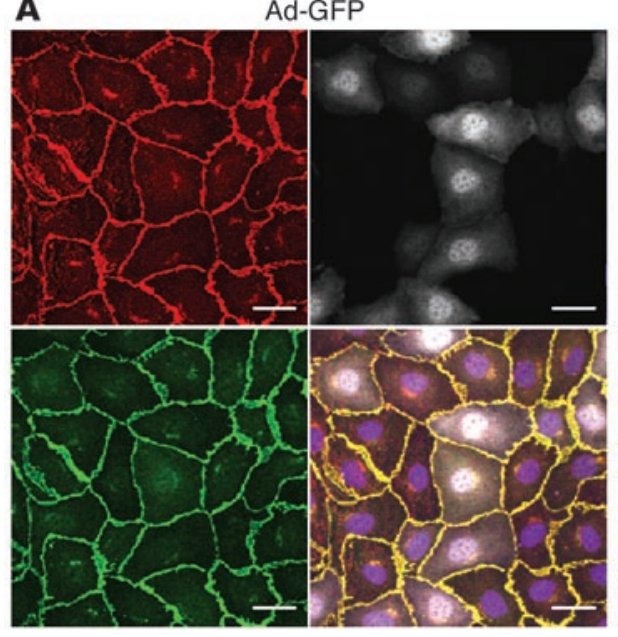

Ad-FGFR1DN

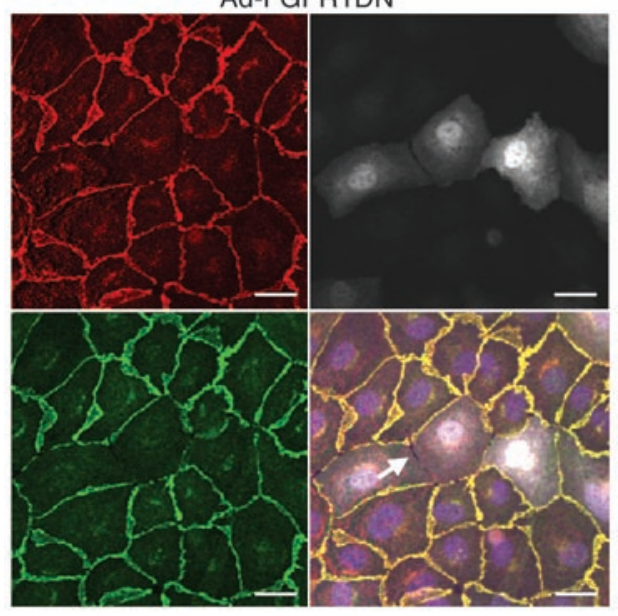

VE-cadherin

$\beta$-catenin

GFP
B

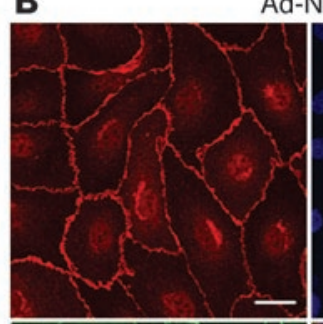

Ad-Null

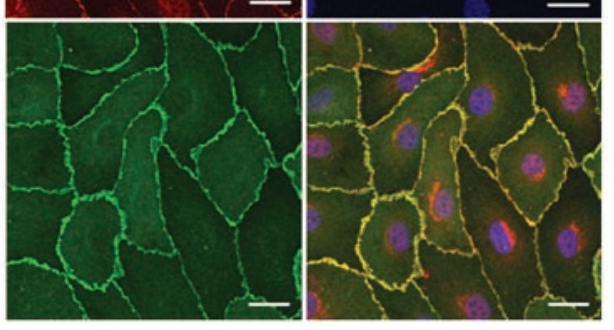

Ad-sFGFR1IIIc
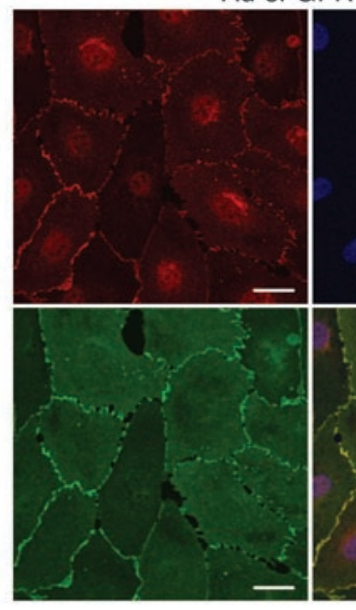
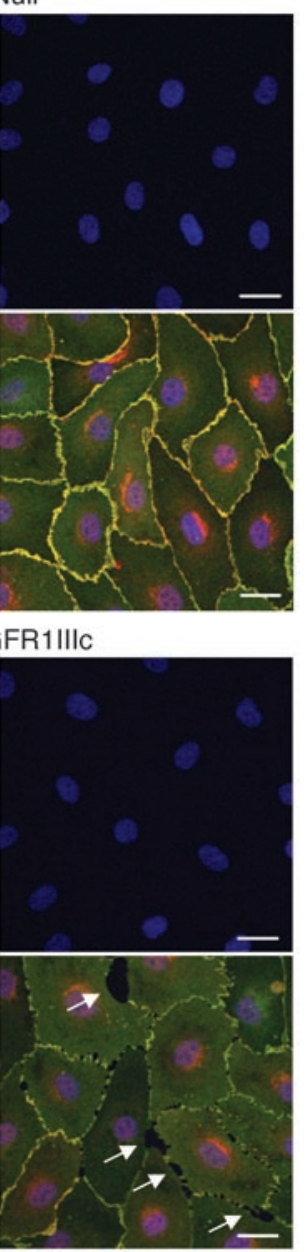

VE-cadherin p120-catenin DAPI

\section{Figure 6}

Junction proteins are absent from cell-cell contacts in cells lacking FGF signaling. (A) Immunostaining of quiescent and fully confluent endothelial monolayer. BAECs were transduced with Ad-GFP or Ad-FGFR1DN-GFP using low MOI (10 and 25, respectively) to limit the number of transduced cells and minimize virusmediated toxicity. Cells were stained for VE-cadherin (red) and $\beta$-catenin (green) 24 hours later. The Ad-FGFR1DN-GFP vector has a bidirectional promoter encoding both GFP and FGFR1DN, thus marking transduced cells with GFP (shown in white). Note the loss of VE-cadherin and $\beta$-catenin staining at cell-cell contacts of FGFR1DNGFP-transduced cells. Arrow points to the gap between neighboring FGFR1DN-GFP-transduced cells. Scale bars: $20 \mu \mathrm{m}$. (B) Immunostaining of quiescent endothelial monolayer (BAECs) transduced with Ad-Null or AdsFGFR1Illc. Cells were stained for VE-cadherin (red), p120catenin (green), and DAPI (blue). sFGFR was secreted in the medium; the effect is not limited to the transduced cells. Arrows indicate gaps between endothelial cells. Scale bars: $20 \mu \mathrm{m}$. they can be compensated for by other FGF family members. This is in agreement with a mild phenotype observed in the $\mathrm{Fgf1}^{-/-} / \mathrm{Fg}^{-1-}$ double knockouts (28). FGF9 knockout results in sex reversal and poor development of lung parenchyma but no vascular defects (29, 30), while FGF20 knockout has not been reported. As sFGFR3IIIc induced effects similar to those observed with sFGFR1IIIc, it is likely that FGF ligands that bind both isoforms, i.e., FGF2, -4, and -8 , are proximal candidates for this vascular integrity maintenance effect. Interestingly, $\mathrm{Fg} \mathrm{fl}^{-/-}$or $\mathrm{Fg} \mathrm{fl}^{-/-}$mice are embryonic lethal, with the $\mathrm{Fg} f 4^{-/-}$embryo being defective in inner cell mass proliferation after implantation and the $F g f 8^{-/-}$embryo showing gastrulation defects $(31,32)$, while $F g f 8$ compound heterozygous mice exhibit defective aortic arch artery formation (33).

The effect of suppression of FGF signaling is similar in arterial and venous vascular beds, implying that ongoing FGF signaling is required equally throughout the vascular system to maintain structural integrity.

As $\mathrm{N}$-cadherin is implicated in mediating mural cell adhesion to endothelial cells (34) and is known to associate with FGFR in cancer cells, thereby modulating FGF signaling activities (35), we investigated whether inhibition of FGF signaling has affected
$\mathrm{N}$-cadherin expression and/or function. We found that neither total N-cadherin expression nor its plasma membrane levels nor its perivascular localization nor endothelial cell attachment to smooth muscle cells were affected by suppression of FGF signaling. Furthermore, since total cellular expression of VE-cadherin was not affected by FGF inhibition, it is unlikely that downregulation of $\mathrm{N}$-cadherin, which has been shown to result in loss of VE-cadherin and p120-catenin expression (26), is the reason for the observed VE-cadherin dysfunction in these settings.

VE-cadherin/p120-catenin/ $\beta$-catenin interaction is the key event in formation of adherens junctions, linking to actin cytoskeleton and regulation of permeability (22). The critical role played by p120-catenin in the cadherins' adhesive function is well established. For the maintenance of normal epithelial morphology, E-cadherin/p120-catenin interaction is essential and cadherinmediated adhesion is impaired as a direct consequence of p120 insufficiency (36). Moreover, p120-catenin controls cadherin turnover, thereby regulating cadherin levels. p120-catenin knockdown by siRNA results in dose-dependent elimination of multiple cadherins including VE-cadherin in endothelial cells and a complete loss of cell-cell adhesion (37). Regulation of VE-cadherin stability 
A

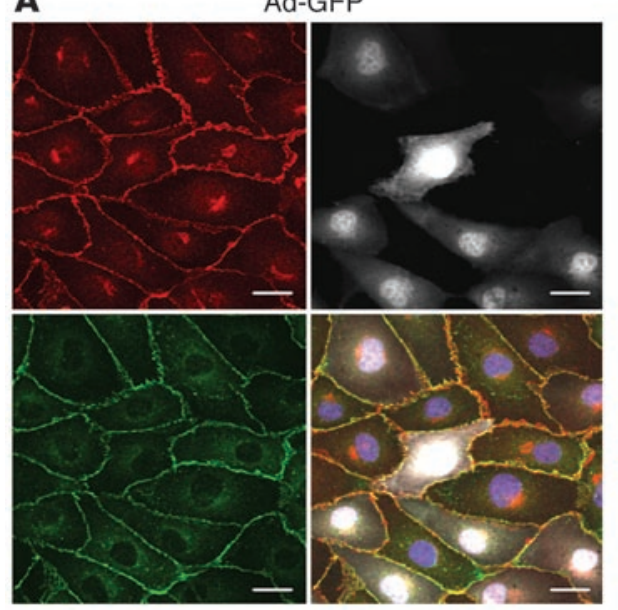

Ad-FGFR1DN
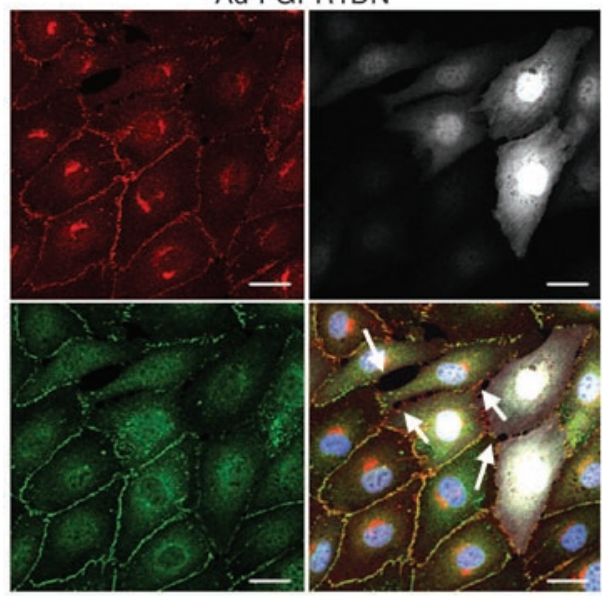

VE-cadherin
ZO-1
GFP
B

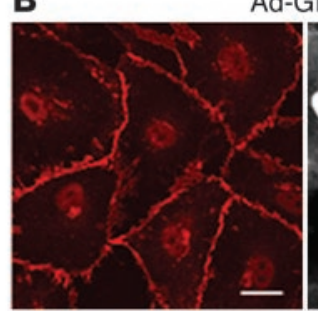

Ad-GFP

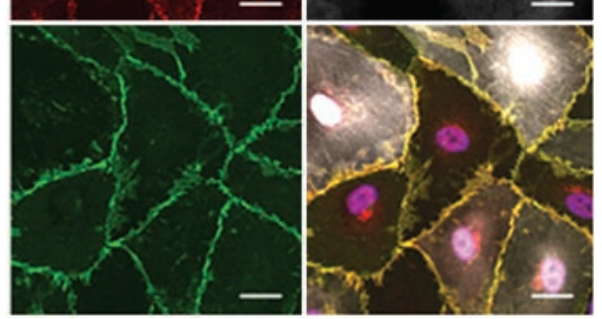

Ad-FGFR1DN

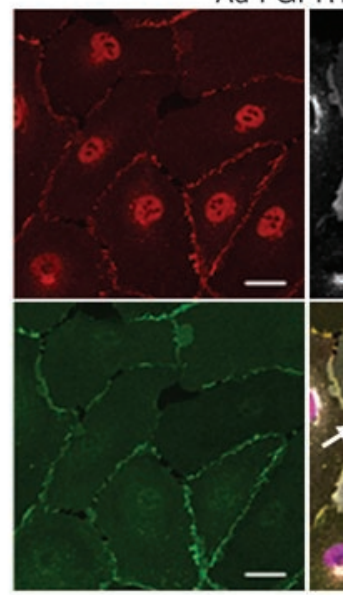

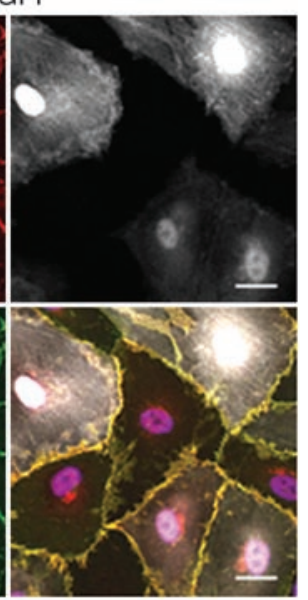

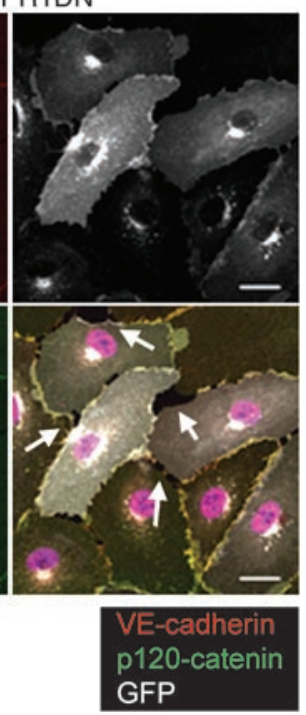

Figure 7

Tight junction and venous cells are similarly affected by FGF signaling inhibition. (A) Immunostaining of quiescent endothelial monolayer (BAECs) transduced with Ad-GFP or AdFGFR1DN-GFP. Cells were stained for VE-cadherin (red) and ZO-1 (green). The Ad-FGFR1DN-GFP vector has a bidirectional promoter encoding both GFP and FGFR1DN as described above (GFP signal is shown in white). Arrows indicate reduced $V E$-cadherin and ZO-1 staining and gaps between endothelial cells. Scale bars: $20 \mu \mathrm{m}$. (B) Immunostaining of quiescent venous endothelial monolayer. HSVECs were transduced with Ad-GFP or Ad-FGFR1DN-GFP and stained for VE-cadherin (red) and p120-catenin (green) 24 hours later. Note loss of VE-cadherin and $\mathrm{p} 120$ catenin staining at cell-cell contacts and formation of intercellular gaps of FGFR1DN-GFP-transduced cells (white arrows). Scale bars: $20 \mu \mathrm{m}$. and internalization is critical to controlling endothelial permeability and barrier function. p120-catenin inhibits VE-cadherin endocytosis via a mechanism that requires direct interactions between p120-catenin and the VE-cadherin juxtamembrane domain (38).

Src activation is an important event in triggering VE-cadherin internalization and adherens junction disruption. VEGF has been known to increase endothelial permeability in an Src-dependent manner by disassembling VE-cadherin-based junctions (39). However, the identity of Src substrate(s) in adherens junctions is still under active debate. In fact, many adherens junction proteins (VE-cadherin, $\beta$-catenin, $\gamma$-catenin/plakoglobin, and p120-catenin) become tyrosine phosphorylated downstream of VEGFR2 upon VEGF stimulation (40), leading to dissociation of endothelial cellcell contacts in vitro. Moreover, recent reports demonstrate that multiple tyrosine residues in the VE-cadherin cytoplasmic tail can be phosphorylated by Src activation under certain experimental conditions (41-43); however, the role and biological relevance of each tyrosine phosphorylation is not firmly established at this point. One interesting observation is that Src inhibitor Csk binds to the phosphorylated $\mathrm{Y}^{685}$ site of VE-cadherin, thus potentially inhibiting Src activity and preventing disruption of the VE-cadherin complex (44). Furthermore, a study demonstrated that a serine residue ( $\left.\mathrm{S}^{665}\right)$ also becomes phosphorylated as a consequence of VEGF-induced Src activation, leading to VE-cadherin internalization (45). On the other hand, p120-catenin, originally identified as an Src substrate, is differentially phosphorylated by Src and Fyn, thus controlling RhoA affinity to p120-catenin and potentially RhoA activity (46). It has been also shown that $\mathrm{p} 190 \mathrm{RhoGAP}$ transiently interacts with p120-catenin, which is required for localized inhibition of Rho and adherens junction formation (47). Therefore, given this complex and poorly understood nature of VE-cadherin regulation, it is difficult to identify the proximal event triggering VE-cadherin/p120-catenin dissociation in the absence of FGF signaling.

Both VEGF and FGF families are potent angiogenic growth factors. However, certain functional differences among blood vessels generated by these factors have long been noted. Whereas VEGFinduced angiogenesis is often accompanied by vascular leakage, FGFs induce growth of nonleaky vessels. At the morphological level, VEGF-induced capillaries have fenestrations, whereas FGF-induced ones are tightly sealed (4). Our data demonstrated that treatment of normal endothelial cells with FGF1 leads to a further increase in VE-cadherin/p120-catenin binding, which may explain the molecular basis of morphological and functional differences of blood vessels generated by these growth factors. Furthermore, the FGF system 

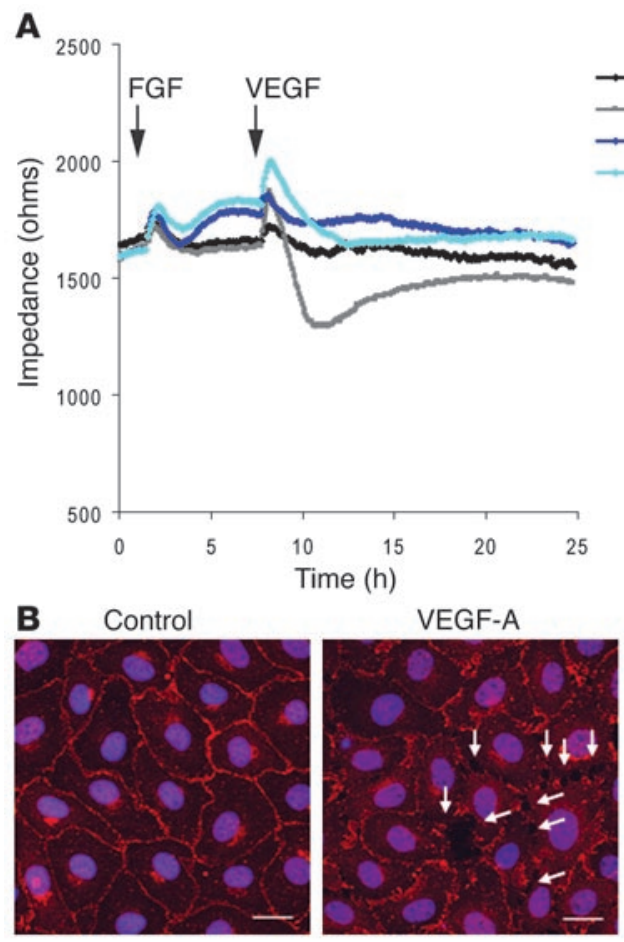

$\rightarrow$ FGF 0 ng/ml, VEGF $0 \mathrm{ng} / \mathrm{ml}$ - FGF $0 \mathrm{ng} / \mathrm{ml}$, VEGF $25 \mathrm{ng} / \mathrm{ml}$ $\rightarrow$ FGF $1 \mathrm{ng} / \mathrm{ml}$, VEGF $0 \mathrm{ng} / \mathrm{ml}$ - FGF $1 \mathrm{ng} / \mathrm{ml}$, VEGF $25 \mathrm{ng} / \mathrm{ml}$

\section{Figure 8}

FGF treatment maintains EC junctions and decreases endothelial permeability in vitro. (A) ECIS permeability assay using confluent monolayer. BAECs are matured to full confluency and the medium was replaced to $1 \%$ BSA in EBM-2 (Cambrex). FGF1 $(1 \mathrm{ng} / \mathrm{ml})$ or $1 \% \mathrm{BSA} / \mathrm{EBM}-2$ was added at the indicated time point (arrow). Six hours later, VEGF-A $(25 \mathrm{ng} / \mathrm{ml})$ or $1 \% \mathrm{BSA} /$ EBM2 was added at the indicated time point (arrow). At 6 hours, FGF treatment significantly increased monolayer impedance compared with the control treatment. (B) FGF treatment does not induce junction disruption on endothelial cell monolayers. BAECs are matured to full confluency, starved with $1 \%$ BSA in EBM-2 for 48 hours, and treated with either FGF1 (50 ng/ml) or VEGF $(80 \mathrm{ng} / \mathrm{ml})$ for 30 minutes Note with this condition, VEGF rapidly disrupts endothelial cell junctions (white arrows) whereas FGF treatment maintains junctions similar to those in the control monolayer. Scale bars: $20 \mu \mathrm{m}$.

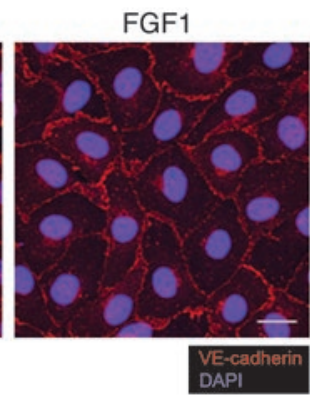

may counteract the VEGF-induced VE-cadherin disassembly, thus playing a critical role in the maintenance of vascular homeostasis.

Thus, our data suggest that FGF-VEGF signaling balance lies at the center of regulation of permeability. At a functional level, this is illustrated by opposite effects of these growth factors on the endothelial monolayer (Figure 8A).

The disassembly of intercellular junctions due to internalization of VE-cadherin from the cell plasma membrane fully explains the phenotype in mice with deficient endothelial FGF signaling. The progressive loss of endothelial cells from the surface of a normal artery exposed to Ad-FGFR1DN is consistent with reduced survival of endothelial cells with a homozygous VE-cadherin deletion and antibody-mediated VE-cadherin disruption $(24,48)$. Furthermore, loss of VE-cadherin cell surface expression has been shown to be critical for disassembly of newly formed blood vessels (49).

In summary, we demonstrate that FGF signaling in endothelial cells is required for the maintenance of endothelial homeostasis and vascular integrity. In the absence of FGF signaling, VE-cadherin undergoes p120-catenin decoupling, leading to the loss of adherens and tight junctions, increased vascular leakiness, and disassembly of the existing vasculature. These results demonstrate the vital role of FGF signaling in maintenance of vascular integrity.

\section{Methods}

Gene construction. The mouse FGFR1 (flg) $\Delta 341-733$ cDNA clone was a gift from A. Mansukhani (New York University, New York, New York, USA). The truncated form of FGFR1 lacking the cytoplasmic part of the receptor (FGFR1DN) encodes the entire extracellular 2 Ig domains with 32 amino acids of the intracellular region (50). To design sFGFR constructs, extracellular parts of mouse FGFR1DN cDNA (FGFR1IIIc isoform), mouse FGFR3IIIb, and mouse FGFR3IIIc cDNA (gift from D. Ornitz, Washington University in St. Louis School of Medicine, St. Louis, Missouri, USA) were amplified by PCR with 5' HindIII and 3' BamHI sites, respectively. After restriction enzyme digestion, PCR products were fused in frame to the hinge- $\mathrm{CH}_{2}-\mathrm{CH}_{3}$ region of human IgG1 (CDM7B-/CD5-Ig expression vector) (20). FGFR1DN and sFGFR cDNAs were subcloned into an adenovirus shuttle vector, and nonreplicative, recombinant adenovirus vectors were generated and propagated to high titer.

Antibodies. Antibodies used for immunoprecipitation, Western blotting, and immunofluorescence include anti-phospho- and total Erk1/2, anti- $\beta$-catenin, and anti-caspase-3 (Cell Signaling Technology), anti-VE-cadherin (Santa Cruz Biotechnology Inc. and Research Diagnostics Inc.), anti-p120-catenin (BD Biosciences - BD Transduction Laboratories), anti-ZO-1 (Invitrogen), $\mathrm{N}$-cadherin (Zymed Laboratories Inc.), and anti- $\beta$-tubulin (Sigma-Aldrich).

Cell culture and adenoviral transduction. BAECs and HSVECs were cultured at $37^{\circ} \mathrm{C}$ in $5 \% \mathrm{CO}_{2}$ in EGM-2 MV medium (Cambrex) or MCDB- 131 complete medium (VEC Technologies Inc.), respectively, and precoated with fibronectin $(10 \mu \mathrm{g} / \mathrm{ml})$ unless otherwise specified. Adenoviral vectors were transduced at an MOI of 10-100 PFUs/cell for Ad-FGFR1DN or 50-200 viral particles/cell for Ad-sFGFRs. The infection medium was replaced 4-6 hours later with the normal growth medium.

Western blotting and immunoprecipitation. The procedure described previously was applied with small modifications (51). In brief, cells were washed twice with ice-cold $\mathrm{Ca}^{2+}$ and $\mathrm{Mg}^{2+} \mathrm{PBS}$ and lysed in RIPA buffer: $50 \mathrm{mM}$ Tris- $\mathrm{HCl}, \mathrm{pH}$ 7.4, $150 \mathrm{mM} \mathrm{NaCl}, 1 \% \mathrm{NP} 40,0.5 \%$ sodium deoxycholate, $0.1 \% \mathrm{SDS}, 2 \mathrm{mM} \mathrm{CaCl}_{2}, 1 \times$ complete protease inhibitor mixture (Roche), and $1 \mathrm{mM}$ sodium orthovanadate. For Erk1/2 phosphorylation studies, to obviate possible quenching of signal that occurs when probing the same protein using different antibodies on the same membrane, the identical samples were run in duplicate and membranes were blotted with either phospho-Erk $1 / 2$ or total-Erk antibody.

For immunoprecipitation, samples were precleared on protein $\mathrm{A} / \mathrm{G}$ beads (Calbiochem) for 1 hour at $4{ }^{\circ} \mathrm{C}$. VE-cadherin was immunoprecipitated from $500 \mu \mathrm{g}$ of extract using $2 \mu \mathrm{g}$ of anti-VE-cadherin antibody followed 
A Ad-GFP Ad-FGFR1DN

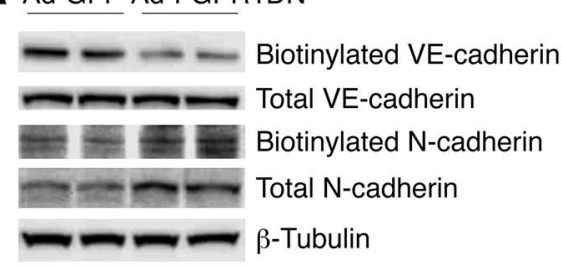

B

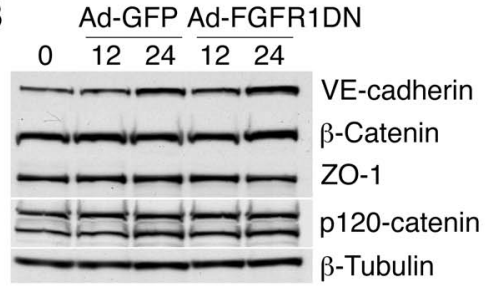

C FGF1: $0 \quad \begin{array}{llllll} & 5 & 15 & 30 & 60 & (\mathrm{~min})\end{array}$

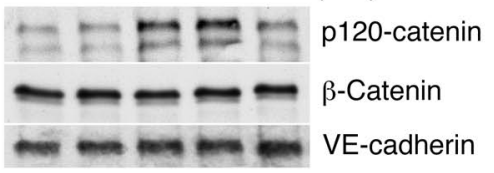

D

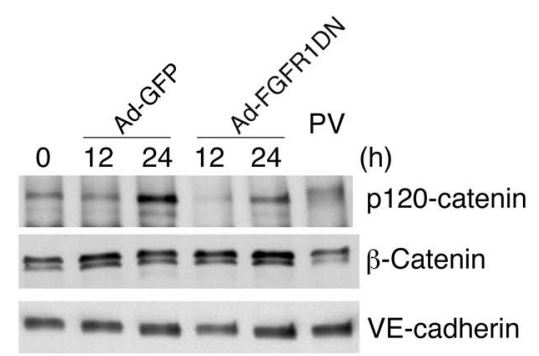

Figure 9

FGF dependence on VE-cadherin/p120-catenin interaction and VE-cadherin plasma membrane retention. (A) Decreased cell surface VE-cadherin in Ad-FGFR1DN-transduced BAECs. Quiescent BAECs were transduced with Ad-GFP or Ad-FGFR1DN and the monolayer was treated with biotin to label cell surface proteins. Biotinylated proteins were subjected to Western blot analysis and probed for VE-cadherin or N-cadherin. Total cell lysates from the same samples were also analyzed by Western blotting to evaluate total protein expression. (B) Western blot analysis of expression of junction proteins. Transduced BAECs were harvested at the indicated time points, and total cell lysates were analyzed by Western blotting. (C) Effect of FGF treatment on VE-cadherin interaction with catenins. BAEC monolayers were maintained in $0.5 \%$ FBS basal medium for 24 hours and then stimulated with FGF1 $(50 \mathrm{ng} / \mathrm{ml})$ for the indicated times. Total cell lysates were immunoprecipitated with antiVE-cadherin antibody and blotted with $\mathrm{p} 120$-catenin, $\beta$-catenin, or VE-cadherin antibody. (D) FGFR signaling requirement for VE-cadherin interaction with catenins. Subconfluent BAEC monolayer ( $70 \%$ confluency) was transduced with Ad-GFP or Ad-FGFR1DN, and cells were grown to form mature junctions. At indicated time points, total cell lysates were prepared and immunoprecipitated with VE-cadherin antibody followed by immunoblotting with $\mathrm{p} 120$-catenin, $\beta$-catenin, or VE-cadherin antibody. As the monolayer forms mature junctions, VE-cadherin/p120-catenin interaction increases (Ad-GFP), whereas this interaction was decreased in Ad-FGFR1DN-treated cells. Nontransduced cells were treated with $100 \mu \mathrm{M}$ PV for 10 minutes prior to cell lysis, showing disrupted VE-cadherin/p120 interaction.

by $20 \mu \mathrm{l}$ of protein $\mathrm{A} / \mathrm{G}$ beads. Beads were washed 5 times using cold PBS, boiled in SDS-PAGE loading buffer, and resolved by SDS-PAGE. Where indicated, cells were treated prior to lysis with $100 \mu \mathrm{M}$ PV for 5 minutes at $37^{\circ} \mathrm{C}$ to inhibit tyrosine phosphatases.

Immunofluorescence. Immunocytochemistry was performed with a standard procedure using antibodies described above. In brief, cells were fixed with $2 \%$ paraformaldehyde and permeabilized with $0.1 \%$ Triton X-100. Incubation with the primary antibody was followed by incubation with Alexa Fluor-conjugated secondary antibodies (Invitrogen). Samples were examined under a Zeiss LSM 510 confocal microscope.

Biotinylation of cell surface protein. Twenty-four hours after adenovirus transduction, cells were washed twice with ice-cold PBS and incubated with $0.5 \mathrm{mg} / \mathrm{ml}$ sulfo- $N$-hydroxysuccinimide ester long-chain biotin (Pierce Biotechnology) in PBS, $\mathrm{pH} 7.4$, for 15 minutes at $4^{\circ} \mathrm{C}$. The biotinylation reaction was quenched with ice-cold $1 \%$ BSA-PBS. Cell lysate was incubated with immobilized NeutrAvidin (Pierce Biotechnology) at $4^{\circ} \mathrm{C}$ to separate biotinylated proteins.

Ad-sFGFR infection and measurement of sFGFR in mouse blood and tissue. All animal experiments were performed under a protocol approved by the Institutional Animal Care and Use Committee of Dartmouth College.

Wild-type male C57BL/ 6 mice or male NU/NU mice (Charles River laboratories) were injected with $5 \times 10^{10}$ viral particles from the tail vein in conjunction with $10 \mu \mathrm{M}$ antennapedia peptide (52) (gift from W.C. Sessa, Yale University School of Medicine, New Haven, Connecticut, USA). Plasma and tissue levels of sFGFRs were measured using a human IgG ELISA quantitation kit (Bethyl Laboratories Inc.).

Permeability assays. $0.5 \%$ Evans blue dye $(200 \mu \mathrm{l})$ was injected into the retroorbital space and was allowed to circulate for 30 minutes. The mice were then sacrificed, blood was drained by perfusion with saline, and the gas- trocnemius muscle, lungs, and heart were excised and dried at $55^{\circ} \mathrm{C}$. Evans blue dye in tissues was extracted with formamide for 24 hours at $55^{\circ} \mathrm{C}$ and its fluorescence at $607 \mathrm{~nm}$ was measured by a fluorescent reader (BioTek).

Transwell tracer experiments with fluorescent dextran were performed using the HTS Fluoroblok 24-well Transwell system (1.0 $\mu \mathrm{m}$ pore; BD). BAECs $\left(2 \times 10^{4}\right)$ were plated on a Transwell insert membrane coated with $10 \mu \mathrm{g} / \mathrm{ml}$ fibronectin. The monolayer was matured until full confluence and transduced with adenoviruses. Thereafter, 2 MDa FITC-dextran was added in the upper chamber and the fluorescence of the lower chamber was measured at fixed time points.

Transendothelial electrical resistance, an index of endothelial cell barrier function, was measured in real time using an electric cell-substrate impedance sensing (ECIS) system (Applied BioPhysics) (53). BAECs $\left(5 \times 10^{4}\right.$ per well) were plated on sterile 8-chambered gold-plated electrode arrays (8W10E) precoated with fibronectin $(10 \mu \mathrm{g} / \mathrm{ml})$ and grown to full confluence. Immediately after adenoviral transduction, the electrode arrays were mounted on the ECIS system within an incubator $\left(37^{\circ} \mathrm{C}, 5 \% \mathrm{CO}_{2}\right)$ and connected to its recorder device. Monolayer resistance was recorded for 50 hours in 5-minute intervals. After 6-hour exposure to adenovirus, viral-containing medium was replaced with normal growth medium (EGM-2 MV).

Adenoviral transduction and enface VE-cadherin staining of artery. Adenovirus $\left(10^{9} \mathrm{PFUs}\right)$ premixed with the $10 \mu \mathrm{M}$ antennapedia peptide (52) was transduced in the rat femoral artery as described previously (54). The artery was excised and cut open longitudinally to expose the endothelium. The arterial segment was placed with the endothelial side up and fixed with tungsten pins (Fine Science Tools). After fixation with $2 \%$ paraformaldehyde in PBS, standard immunostaining was performed using anti-VE-cadherin (Santa Cruz Biotechnology Inc.) and Alexa Fluor 568-conjugated anti-goat antibody (Invitrogen). Using a Zeiss LSM 510 confocal microscope, 20-30 Z-Stack 
$1-\mu \mathrm{m}$ optical sections were acquired and projected to a single image plane. To examine the effect of sFGFR, wild-type mice were infected $\left(5 \times 10^{10}\right.$ viral particles with $10 \mu \mathrm{M}$ antennapedia) and the carotid artery and jugular vein were stained for VE-cadherin and visualized as described above.

Mouse trachea vasculature studies. Sites of leakage in mouse tracheal blood vessels were visualized using $100 \mathrm{~nm}$ extravasated green fluorescent polymer microspheres (Duke Scientific Corp.) as described previously (55). In brief, 10 days after injection of control or sFGFR1IIIc adenovirus, anesthetized mice received $20 \mu \mathrm{l}\left(4 \times 10^{11}\right.$ particles $)$ i.v. injection of fluorescent microspheres. Two minutes later, intravascular microspheres were removed from the bloodstream by perfusion of $1 \%$ paraformaldehyde in PBS for 2 minutes at a pressure of $120 \mathrm{mmHg}$. Tracheas were removed from mice and immersion fixed for an additional 2 hours, and then the vasculature was incubated with anti-CD31 antibody (Chemicon) and subsequently with Cy3-conjugated goat anti-hamster IgG (Jackson ImmunoResearch). Trachea whole mounts were examined using a Zeiss LSM 510 confocal microscope.

Electron microscopy: cells. BAECs were grown to confluence on fibronectincoated 12-mm glass coverslips at which point they were transduced with either Ad-GFP or Ad-FGFR1DN-GFP as described. After 24 hours, the cells were fixed (30 minutes, room temperature [RT]) in $2 \%$ glutaraldehyde in $0.1 \mathrm{M}$ sodium cacodylate, rinsed $(2 \times 10$ minutes, $\mathrm{RT})$ in $0.1 \mathrm{M}$ sodium cacodylate, and postfixed ( 1 hour, $\mathrm{RT}$, in dark) in $1 \% \mathrm{OsO}_{4}$ in $0.1 \mathrm{M}$ sodium cacodylate. The cells were dehydrated in graded ethanols, critical point dried using a Samdri-795 Critical Point Dryer (Tousimis), coated with a 3-nm amorphous osmium layer in an OPC-60 Osmium Plasma Coater (Filgen), mounted on stubs, and examined at high voltage in an XL-30 ESEM-FEG microscope (FEI Co.) using secondary electron detection.

Electron microscopy: artery specimens. The right femoral artery of male rats was used for epigenetic expression via local adenoviral delivery, as described above. The femoral artery segments that were treated with either Ad-Null or Ad-FGFR1DN as well as the contralateral control segments were removed, rinsed in PBS, and cut open longitudinally. The artery fragments were opened flat with the endothelium facing upwards, pinned down using fine tungsten pins, and fixed ( 1 hour, RT) in $2 \%$ glutaraldehyde. At all times during these procedures, the tissue was submerged in liquid. The arteries were then processed for electron microscopy as described for the cells.

Statistics. Statistical analysis was carried out using a 2-tailed Student's $t$ test. Results were considered significant at $P<0.05$.

\section{Acknowledgments}

This work has been supported by NIH grants HL-53793 (to M. Simons) and HL-65418 (to R.V. Stan). We would like to thank Amy Hall and Samantha Shipman (Dartmouth Medical School) for technical assistance and mouse handling and Kenneth Orndorff at the Englert Cell Analysis Laboratory (Dartmouth Medical School) for technical assistance for the confocal microscope system. We would like to thank Frank Giordano and Reed Hickey (Yale University School of Medicine) for adenovirus generation.

Received for publication February 11, 2008, and accepted in revised form July 30, 2008.

Address correspondence to: Michael Simons, Section of Cardiovascular Medicine, Yale University School of Medicine, 330 Cedar Street, PO Box 208017, New Haven, Connecticut 065208017, USA. Phone: (203) 785-7000; Fax: (203) 785-7144; E-mail: michael.simons@yale.edu.

Miasahiro Murakami and Michael Simons's present address is: Section of Cardiovascular Medicine, Department of Internal Medicine, Yale University School of Medicine, New Haven, Connecticut, USA.

Loc T. Nguyen's present address is: Department of Internal Medicine, Yale University School of Medicine, New Haven, Connecticut, USA.
1. Baffert, F., et al. 2006. Cellular changes in normal blood capillaries undergoing regression after inhibition of VEGF signaling. Am. J. Physiol. Heart Circ Physiol. 290:H547-H559.

2. Kamba, T., et al. 2006. VEGF-dependent plasticity of fenestrated capillaries in the normal adult microvasculature. Am. J. Physiol. Heart Circ. Physiol. 290:H560-H576.

3. Karsan, A., et al. 1997. Fibroblast growth factor-2 inhibits endothelial cell apoptosis by Bcl-2-dependent and independent mechanisms. Am. J. Pathol. 151:1775-1784.

4. Cao, R., et al. 2004. Comparative evaluation of FGF-2-, VEGF-A-, and VEGF-C-induced angiogenesis, lymphangiogenesis, vascular fenestrations, and permeability. Circ. Res. 94:664-670.

5. Ornitz, D.M., and Itoh, N. 2001. Fibroblast growth factors. Genome Biol. 2:REVIEWS3005.

6. Presta, M., et al. 2005. Fibroblast growth factor/ fibroblast growth factor receptor system in angiogenesis. Cytokine Growth Factor Rev. 16:159-178.

7. Poole, T.J., Finkelstein, E.B., and Cox, C.M. 2001. The role of FGF and VEGF in angioblast induction and migration during vascular development. Dev. Dyn. 220:1-17.

8. Ortega, S., Ittmann, M., Tsang, S.H., Ehrlich, M., and Basilico, C. 1998. Neuronal defects and delayed wound healing in mice lacking fibroblast growth factor 2. Proc. Natl. Acad. Sci. U. S. A. 95:5672-5677.

9. Grose, R., and Dickson, C. 2005. Fibroblast growth factor signaling in tumorigenesis. Cytokine Growth Factor Rev. 16:179-186.

10. Bottcher, R.T., and Niehrs, C. 2005. Fibroblast growth factor signaling during early vertebrate development. Endocr. Rev. 26:63-77.
11. Eswarakumar, V.P., Lax, I., and Schlessinger, J. 2005. Cellular signaling by fibroblast growth factor receptors. Cytokine Growth Factor Rev. 16:139-149.

12. Ornitz, D.M., et al. 1996. Receptor specificity of the fibroblast growth factor family. J. Biol. Chem. 271:15292-15297.

13. Zhang, X., et al. 2006. Receptor specificity of the fibroblast growth factor family. The complete mammalian FGF family. J. Biol. Chem. 281:15694-15700.

14. Nakamura, T., Mochizuki, Y., Kanetake, H., and Kanda, S. 2001. Signals via FGF receptor 2 regulate migration of endothelial cells. Biochem. Biophys. Res. Commun. 289:801-806.

15. Lavine, K.J., et al. 2006. Fibroblast growth factor signals regulate a wave of Hedgehog activation that is essential for coronary vascular development. Genes Dev. 20:1651-1666.

16. Ueno, H., Gunn, M., Dell, K., Tseng, A., Jr., and Williams, L. 1992. A truncated form of fibroblast growth factor receptor 1 inhibits signal transduction by multiple types of fibroblast growth factor receptor. J. Biol. Chem. 267:1470-1476.

17. Compagni, A., Wilgenbus, P., Impagnatiello, M.A., Cotten, M., and Christofori, G. 2000. Fibroblast growth factors are required for efficient tumor angiogenesis. Cancer Res. 60:7163-7169.

18. Gerber, H.P., et al. 1999. VEGF couples hypertrophic cartilage remodeling, ossification and angiogenesis during endochondral bone formation. Nat. Med. 5:623-628.

19. Rupnick, M.A., et al. 2002. Adipose tissue mass can be regulated through the vasculature. Proc. Natl. Acad. Sci. U. S. A. 99:10730-10735.

20. Bennett, K.L., et al. 1997. Deleted in colorectal carcinoma (DCC) binds heparin via its fifth fibronectin type III domain. J. Biol. Chem. 272:26940-26946.

21. Khurana, R., et al. 2004. Angiogenesis-dependent and independent phases of intimal hyperplasia. Circulation. 110:2436-2443.

22. Dejana, E. 2004. Endothelial cell-cell junctions: happy together. Nat. Rev. Mol. Cell Biol. 5:261-270.

23. Behrens, J., Birchmeier, W., Goodman, S.L., and Imhof, B.A. 1985. Dissociation of Madin-Darby canine kidney epithelial cells by the monoclonal antibody anti-arc-1: mechanistic aspects and identification of the antigen as a component related to uvomorulin. J. Cell Biol. 101:1307-1315.

24. Corada, M., et al. 1999. Vascular endothelial-cadherin is an important determinant of microvascular integrity in vivo. Proc. Natl. Acad. Sci. U. S. A. 96:9815-9820.

25. Mirzapoiazova, T., Kolosova, I., Usatyuk, P.V., Natarajan, V., and Verin, A.D. 2006. Diverse effects of vascular endothelial growth factor on human pulmonary endothelial barrier and migration. Am. J. Physiol. Lung Cell Mol. Physiol. 291:L718-L724.

26. Luo, Y., and Radice, G.L. 2005. N-cadherin acts upstream of VE-cadherin in controlling vascular morphogenesis. J. Cell Biol. 169:29-34.

27. Kowalczyk, A.P., and Reynolds, A.B. 2004. Protecting your tail: regulation of cadherin degradation by p120-catenin. Curr. Opin. Cell Biol. 16:522-527.

28. Miller, D.L., Ortega, S., Bashayan, O., Basch, R., and Basilico, C. 2000. Compensation by fibroblast growth factor 1 (FGF1) does not account for the mild phenotypic defects observed in FGF2 null mice. Mol. Cell. Biol. 20:2260-2268.

29. Colvin, J.S., Green, R.P., Schmahl, J., Capel, B., and Ornitz, D.M. 2001. Male-to-female sex reversal in mice lacking fibroblast growth factor 9. Cell. 
39. Weis, S.M., and Cheresh, D.A. 2005. Pathophysiological consequences of VEGF-induced vascular permeability. Nature. 437:497-504.

Colvin, J.S., White, A.C., Pratt, S.J., and Ornitz, D.M. 2001. Lung hypoplasia and neonatal death in Fgf9-null mice identify this gene as an essential regulator of lung mesenchyme. Development. 128:2095-2106.

31. Feldman, B., Poueymirou, W., Papaioannou, V.E., DeChiara, T.M., and Goldfarb, M. 1995. Requirement of FGF-4 for postimplantation mouse development. Science. 267:246-249.

32. Sun, X., Meyers, E.N., Lewandoski, M., and Martin, G.R. 1999. Targeted disruption of Fgf8 causes failure of cell migration in the gastrulating mouse embryo. Genes Dev. 13:1834-1846.

33. Abu-Issa, R., Smyth, G., Smoak, I., Yamamura, K., and Meyers, E.N. 2002. Fgf8 is required for pharyngeal arch and cardiovascular development in the mouse. Development. 129:4613-4625.

34. Gerhardt, H., Wolburg, H., and Redies, C. 2000. $\mathrm{N}$-cadherin mediates pericytic-endothelial interaction during brain angiogenesis in the chicken. Dev. Dyn. 218:472-479.

35. Suyama, K., Shapiro, I., Guttman, M., and Hazan, R.B. 2002. A signaling pathway leading to metastasis is controlled by $\mathrm{N}$-cadherin and the FGF receptor. Cancer Cell. 2:301-314.

36. Ireton, R.C., et al. 2002. A novel role for $\mathrm{p} 120$ catenin in E-cadherin function. J. Cell Biol. 159:465-476.

37. Davis, M.A., Ireton, R.C., and Reynolds, A.B. 2003. A core function for $\mathrm{p} 120$-catenin in cadherin turnover. J. Cell Biol. 163:525-534.

38. Xiao, K., et al. 2005. p120-Catenin regulates clathrin-dependent endocytosis of VE-cadherin. Mol Biol. Cell. 16:5141-5151.
0. Esser, S., Lampugnani, M.G., Corada, M., Dejana, E., and Risau, W. 1998. Vascular endothelial growth factor induces VE-cadherin tyrosine phosphorylation in endothelial cells. J. Cell Sci. 111:1853-1865.

41. Potter, M.D., Barbero, S., and Cheresh, D.A. 2005. Tyrosine phosphorylation of VE-cadherin prevents binding of $\mathrm{p} 120$ - and beta-catenin and maintains the cellular mesenchymal state. J. Biol. Chem. 280:31906-31912.

42. Wallez, Y., et al. 2006. Src kinase phosphorylates vascular endothelial-cadherin in response to vascular endothelial growth factor: identification of tyrosine 685 as the unique target site. Oncogene. 26:1067-1077.

43. Turowski, P., et al. 2008. Phosphorylation of vascular endothelial cadherin controls lymphocyte emigration. J. Cell Sci. 121:29-37.

44. Baumeister, U., et al. 2005. Association of Csk to VE-cadherin and inhibition of cell proliferation. EMBO J. 24:1686-1695.

45. Gavard, J., and Gutkind, J.S. 2006. VEGF controls endothelial-cell permeability by promoting the betaarrestin-dependent endocytosis of VE-cadherin. Nat. Cell Biol. 8:1223-1234.

46. Castano, J., et al. 2007. Specific phosphorylation of p120-catenin regulatory domain differently modulates its binding to RhoA. Mol. Cell. Biol. 27:1745-1757.

47. Wildenberg, G.A., et al. 2006. p120-Catenin and p190RhoGAP regulate cell-cell adhesion by coordinating antagonism between Rac and Rho. Cell.
127:1027-1039

48. Carmeliet, P., et al. 1999. Targeted deficiency or cytosolic truncation of the VE-cadherin gene in mice impairs VEGF-mediated endothelial survival and angiogenesis. Cell. 98:147-157.

49. Crosby, C.V., et al. 2005. VE-cadherin is not required for the formation of nascent blood vessels but acts to prevent their disassembly. Blood. 105:2771-2776

50. Li, Y., Basilico, C., and Mansukhani, A. 1994. Cell transformation by fibroblast growth factors can be suppressed by truncated fibroblast growth factor receptors. Mol. Cell. Biol. 14:7660-7669.

51. Lampugnani, M.G., et al. 1997. Cell confluence regulates tyrosine phosphorylation of adherens junction components in endothelial cells. J. Cell Sci. 110:2065-2077.

52. Gratton, J.P., et al. 2003. Cell-permeable peptides improve cellular uptake and therapeutic gene delivery of replication-deficient viruses in cells and in vivo. Nat. Med. 9:357-362.

53. Lee, J.F., et al. 2006. Dual roles of tight junction-associated protein, zonula occludens-1, in sphingosine 1-phosphate-mediated endothelial chemotaxis and barrier integrity. J. Biol. Chem. 281:29190-29200.

54. Partovian, C., et al. 2005. PKCalpha activates eNOS and increases arterial blood flow in vivo. Circ. Res. 97:482-487.

55. Baffert, F., Le, T., Thurston, G., and McDonald, D.M. 2006. Angiopoietin-1 decreases plasma leakage by reducing number and size of endothelial gaps in venules. Am. J. Physiol. Heart Circ. Physiol. 290:H107-H118. 